\title{
Ueber die Spaltung der Gelatine.
}

\author{
Von \\ P. A. Levene. \\ Erste Mittheilung.
}

\begin{abstract}
(Aus der physiologisch-chemischen Abtheilung des pathologischen Instituts der New-Yorker Staatskrankenhäuser.)

(Der Redaction zugegangen am 15. Oktober 1902.)
\end{abstract}

\section{Der Glycocollgehalt der Gelatosen.}

Ueber die quantitative Analyse der Spaltungsproducte der verschiedenen Proteosen liegen nur wenige Arbeiten vor, obgleich solche Untersuchungen für die Erklärung der chemischen Vorgänge bei der Verdauung durch proteolytische Enzyme, ebenso wie für die Erklärung des Aufbaues der verschiedenen Eiweisskörper von grosser Wichtigkeit sind. Die Arbeiten von Haslam und Hart sind meines Wissens die ersten auf diesem Gebiete und die letztere erschien, als meine Untersuchungen schon im Gange waren.

Meine Untersuchungen bezweckten, ebenso wie die von Haslam und Hart, die Lösung der Frage, ob Eiweisskörper bei proteolytischer Verdauung in verschiedenartige Theile zerfallen. Jedoch wählte ich als Versuchsmaterial den Leim, da dieser viel Glycocoll liefert, welches sich ja nach dem Verfahren von E. Fischer genau quantitativ bestimmen lässt.

In einer kürzlich erschienenen Mittheilung hat E. Fischer erwiesen, dass die Zersetzungsproducte des Caseins die Eigenschaft besitzen, die vollständige Ausscheidung des salzsauren Glycocollesters zu verhindern. Aus einer Mischung der Spaltungsproducte des Caseins liess sich nur etwa 75\% des zugesetzten Glycocolls gewinnen. Es war nur nothwendig, festzustellen, wie sich in dieser Beziehung die Gelatine bei der Zersetzung verhält. 
$\mathrm{Zu}$ diesem Zweck wurden $50 \mathrm{~g}$ Leim in üblicher Weise mit Salzsäure hydrolysirt und das so erhaltene Product mit absolutem Alkohol und trockner Salzsäure verestert. Das Verfahren wurde nach Fischer's Methode dreimal wiederholt, schliesslich über Nacht stehen gelassen, dann wieder zur Consistenz eines Oels in Vacuo eingedampft, und darauf in eine Kältemischung eingetragen. Bei dieser Behandlung fing die Krystallisation des Glycocollesters in kurzer Zeit an, oft auch ohne Impfung mit einem Krystall des Esters. Um eine möglichst vollständige Ausscheidung des salzsauren Glycocollesters zu erzielen, wurde die Mischung im Eisschrank etwa 48 Stunden gelassen. Die Mutterlauge von diesem Salze gab keine neue Ausscheidung desselben mehr, selbst nicht nach monatelangem Stehen im Eisschranke.

$\mathrm{Zu}$ einer derartigen Mutterlauge wurden 8,5 g Glycocoll eingetragen und die Mischung auf übliche Weise dreimal verestert. Es gelang bei dieser Behandlung, 14,5 g des Glycocollesters wieder zu gewinnen. 91,64\% des zugefügten Glycocolls wurden also wieder gefunden.

Diese Methode der Glycocollbestimmung schien also befriedigend für ein vergleichendes Studium, hauptsächlich wenn die Quantitäten des angewandten Materials und die anderen Verhältnisse des Experiments gleich blieben.

Um die Vorgänge bei peptischer, tryptischer und PapainVerdauung $\mathrm{zu}$ vergleichen, wurden die Pepto- wie die Tryptound Papaio-Gelatosen in gleicher Weise analysirt.

\section{Darstellung der Gelatosen.}

Trennung der Proto- und Deuterogelatose.

Die Gelatosen wurden erhalten durch fractionirte Fällung der Verdauungsproducte mit Hülfe von schwefelsaurem Ammon. Die neutralisirten Verdauungsproducte wurden eingedampft und mit einem gleichen Volumen kaltgesättigter Lösung des Salzes behandelt. Den auf diese Weise resultirenden Niederschlag löste ich in Wasser und dialysirte ihn, bis er frei von Salz geworden war. Diese Lösung, wieder mit einem gleichen 
Volumen gesättigtèr Ammoniumsulfatlösung vermischt, gab einen Niederschlag, der als Fraction I betrachtet werden wird, und der thatsächlich aus Protogelatosen bestand. Die Gelatosen wurden vom Salz durch Dialyse befreit, die salzfreie Lösung bis zu der Consistenz eines dicken Syrups eingedampft und in absoluten Alkohol eingetragen. Den hierbei entstehenden Niederschlag musste man möglichst schnell filtriren und sofort im Vacuum über Schwefelsäure trocknen. Der vacuum-trockne Niederschlag konnte dann ohne Zersetzung auf höhere Temperaturen erhitzt werden, wenn man die Temperatursteigerung gan\% allmählich vornahm. Die Gelatosen wurden schliesslich bei $110^{\circ}$ C. getrocknet.

Das Filtrat vom ersten Niederschlage (Fraction I) wurde mit schwefelsaurem Ammon gesättigt, der dabei ausfallende Niederschlag in Wasser aufgenommen und dialysirt, bis das Salz vollständig entfernt war. Die salżfeie Lösung wurde wieder mit einem gleichen Volumen kalt gesättigter Ammoniumsulfatlösung versetzt, filtrirt und aus dem Filtrate durch Sättigen mit demselben Salze die zweite Fraction erhalten, welche aus Deuteroalbumosen bestand. Die Reinigung und das Trocknen dieser Fraction wurde in derselben Weise wie bei der ersten Fraction ausgeführt.

\section{Trypsin-Verdauung.}

$500 \mathrm{~g}$ französische Gelatine (Gold-Marke) wurden in 8 Liter $0,5 \%$ iger Natriumcarbonatlösung aufgenommen und mit 10\% Trypsin (Trypsinum purissimum Grübler) der Verdauung bei Zimmertemperatur überlassen. Als Antisepticum wurde Toluol hinzugefügt. Nach 48 Stunden wurde die Verdauung abgebrochen, da eine dann entnommene Probe, nachdem sie neutralisirt und bis zur Hälfte eingedampft war, nicht mehr gelatinirte. Das ganze Verdauungsproduct wurde dann neutralisirt, filtrirt und aus dem eingedampften Filtrate die Gelatosen gewonnen.

\section{Pepsin-Verdauung.}

$500 \mathrm{~g}$ derselben Gelatine in 8 Liter $0,2 \%$ iger wässeriger Salzsäure aufgenommen und mit $5 \mathrm{~g}$ Pepsin (Fairchild Scales) 
nach Toluolzusatz eine Woche der Verdauung überlassen, da eine eingedampfte, neutralisirte Probe erst dann das Gelatinirungsvermögen eingebüsst hatte.

\section{Papain-Verdauung.}

$800 \mathrm{~g}$ Gelatine in 8 Liter 0,5\% iger Natroncarbonatlösung aufgenommen und mit $10 \mathrm{~g}$ Papain (Mera) zwei Wochen der Verdauung überlassen. Die Trennung der Albumosen wurde in der oben beschriebenen Weise mit schwefelsaurem Ammon ausgeführt.

Da keine Analyse der Papaio-Gelatosen vorliegt, so wurden die Präparate auf ihren Gehalt an $\mathrm{C}$ und $\mathrm{H}$ untersucht.

$0,2860 \mathrm{~g}$ trockner und aschefreier Papaio-Gelatose ergaben bei Verbrennung $0,5288 \mathrm{CO}_{2}$ und $0,1705 \mathrm{H}_{2} \mathrm{O}$; also $\mathrm{C}=50,43 \%$ und $\mathrm{H}=6,63$.

$0,3345 \mathrm{~g}$ Deutero-Papaio-Gelatose ergaben $0,6063 \mathrm{~g} \mathrm{CO}_{2}$ und $0,2084 \mathrm{H}_{2} \mathrm{O}$; also $\mathrm{C}=50,00 \%$ und $\mathrm{H}=6,83 \%$.

Diese Zahlen stimmen mit denen von Chittenden und Solly für andere Gelatine selbst vollkommen überein.

\begin{tabular}{lccccccccc} 
& \multicolumn{3}{c}{ Proto-Gelatosen } & \multicolumn{3}{c}{ Deutero-Gelatosen } \\
& C & H & N & S & C & H & N & S \\
Gelatine & 49,00 & 6,81 & 17,97 & 0,71 & - & - & - & - & ('iittenden, Solly \\
Pepto-Gelatine & 49,98 & 6,78 & 17,86 & 0,52 & 49,23 & 6,84 & 17,40 & 0,57 & $:$ \\
Trypto-Gelatine & 49,45 & 6,81 & 17,87 & 0,57 & 49,07 & 6,66 & 17,52 & 0,65 & : \\
Papaio-Gelatine & 50,43 & 6,63 & - & - & 50,00 & 6,83 & - & - Levene.
\end{tabular}

\section{Unverdaute Gelatine.}

Für ein Experiment wurde die Gelatine bei $120^{\circ}$ C. bis zu constantem Gewicht getrocknet. Bei einem anderen Versuche wurde sie nach Chittenden gereinigt.

$500 \mathrm{~g}$ derselben Gelatine wurden in etwas Wasser gelöst und die Lösung in absoluten Alkohol gegossen. Es entstand dabei ein gummiartiger Niederschlag, der sich durch Abpressen theilweise vom Alkohol befreien liess. Er wurde nun in Vacuo über Schwefelsäure getrocknet und dann in ein Luftbad gebracht, wo er unter allmählicher Erhöhung der Temperatur auf $110^{\circ}$ der Trocknung überlassen wurde.

\section{Spaltung und Bestimmung des Glycocolls.}

Die Gelatine oder die Gelatosen wurden in der dreifachen Quantität Salzsäure (specifisches Gewicht 1,20) gelöst und dann 
6 Stunden am Rückflusskühler gekocht. Das Verestern und Krystallisiren wurde nach Fischer's Methode, wie oben angegeben, ausgeführt. Um die Reste des Glycocolls aus der Mutterlauge zu gewinnen, wurden aus der letzteren die freien Aminosäuren nach Fischer's Verfahren dargestellt, und die Fraction unter $75^{\circ} \mathrm{C}$. wieder mit Alkohol und getrockneter Salzsäure behandelt. Es schieden sich bei längerem Stehen nur ganz kleine Quantitäten des Glycocollesters ab.

Die Zahlen, die auf diese Weise für den Salzsäureglycocollester gewonnen waren, sind die folgenden.

I. Gelatine getrocknet bei $120^{\circ}$ C., $50 \mathrm{~g}$ gaben $15,2 \mathrm{~g}$.

II. Gelatine nach Chittenden gereinigt, $49 \mathrm{~g}$ gaben $14,9 \mathrm{~g}$.

III. Proto-Tryptogelatosen, $34 \mathrm{~g}$ gaben $10,8 \mathrm{~g}$ des Hydrochlorats.

IV. Deutero-Tryptogelatosen, $50 \mathrm{~g}$ gaben $18,8 \mathrm{~g}$ des Hydrochlorats.

V. Proto-Peptogelatosen, $50 \mathrm{~g}$ gaben $17,1 \mathrm{~g}$ des Hydrochlorats.

VI. Deutero-Peptogelatosen, $50 \mathrm{~g}$ gaben $18,6 \mathrm{~g}$ des Hydrochlorats.

VII. Papaio-Protogelatosen, $50 \mathrm{~g}$ gaben $18,6 \mathrm{~g}$ des Hydrochlorats.

VIII. Deutero-Papaiogelatosen, $50 \mathrm{~g}$ gaben $18,0 \mathrm{~g}$ des Hydrochlorats.

In der folgenden Tabelle sind die Zahlen für das freie Glycocoll zusammengestellt.

$$
\begin{aligned}
& \text { Gelatine. } \\
& \text { I - }-16,43 \% \\
& \text { II - } 16,34 \% \\
& \text { Proto-Peptogelatose } \quad 18,36 \% \text { Deutero-Peptogelatose } 19,96 \% \\
& \text { - Trypto } 17,07 \% \text { "Trypto > } 20,29 \% \\
& \text { - Papaio > 20,29\% Papaio > 19,33\%. }
\end{aligned}
$$

Aus dieser Tabelle ist es ersichtlich, dass die Gelatosen einen grösseren Gehalt an Glycocoll hatten, als die Gelatine. Der Befund, dass die verschiedenen Protogelatosen in ihrem Gehalt an Glycocoll sich änderten, kann dadurch erklärt werden, dass die Verdauungszeit in den Experimenten variirte.

\section{Litteratur.}

Chittenden und Solly, Journal of Physiology 1891.

Emil Fischer, Diese Zeitschrift, Bd. XXXV.

Haslam, Diese Zeitschrift, Bd. XXXII.

Har t, Diese Zeitschrift, Bd. XXXIII. 